University of Nebraska - Lincoln

DigitalCommons@University of Nebraska - Lincoln

2006

\title{
Atomic force microscope tip spontaneous retraction from dielectric surfaces under applied electrostatic potential
}

\author{
S. F. Lyuksyutov \\ University of Akron \\ P. B. Paramonov \\ University of Akron \\ O. V. Mayevska \\ University of Akron \\ M. A. Reagan \\ University of Akron \\ E. Sancaktar \\ University of Akron \\ See next page for additional authors
}

Follow this and additional works at: https://digitalcommons.unl.edu/usafresearch

Part of the Aerospace Engineering Commons

Lyuksyutov, S. F.; Paramonov, P. B.; Mayevska, O. V.; Reagan, M. A.; Sancaktar, E.; Vaia, R. A.; and Juhl, S., "Atomic force microscope tip spontaneous retraction from dielectric surfaces under applied electrostatic potential" (2006). U.S. Air Force Research. 25.

https://digitalcommons.unl.edu/usafresearch/25

This Article is brought to you for free and open access by the U.S. Department of Defense at DigitalCommons@University of Nebraska - Lincoln. It has been accepted for inclusion in U.S. Air Force Research by an authorized administrator of DigitalCommons@University of Nebraska - Lincoln. 


\section{Authors}

S. F. Lyuksyutov, P. B. Paramonov, O. V. Mayevska, M. A. Reagan, E. Sancaktar, R. A. Vaia, and S. Juhl 


\title{
Atomic force microscope tip spontaneous retraction from dielectric surfaces under applied electrostatic potential
}

\author{
S.F. Lyuksyutov ${ }^{\mathrm{a}, *}$, P.B. Paramonov ${ }^{\mathrm{a}, 1}$, O.V. Mayevska ${ }^{\mathrm{a}}$, M.A. Reagan ${ }^{\mathrm{a}}$, \\ E. Sancaktar ${ }^{\mathrm{a}}$, R.A. Vaia ${ }^{\mathrm{b}}$, S. Juhl ${ }^{\mathrm{b}}$ \\ ${ }^{a}$ Departments of Physics, and Polymer Engineering, The University of Akron, Akron OH 44325, USA \\ ${ }^{\mathrm{b}}$ Materials and Manufacturing Directorate, Air Force Research Laboratory, Wright-Patterson Air Force Base, OH 45433, USA
}

Received 3 November 2005; received in revised form 27 March 2006; accepted 5 April 2006

\begin{abstract}
A time-resolved method for tip' retraction at $\mu$ s-scale away from dielectric surfaces has been developed. Analysis of the forces in the system comprising AFM tip, water meniscus, and polymer film suggests that an electrostatic repulsion of the tip from the surface in the double-layered (water and polymer) system, and water condensation in the tip-surface junction are the dominant factors enabling the mechanical work for tip retraction. Nanostructures of 5-80 nm height are formed in polymeric surfaces as a result. This interesting physical phenomenon could be used for nanostructures patterning in polymeric materials at enhanced aspect ratio.
\end{abstract}

(C) 2006 Elsevier B.V. All rights reserved.

PACS: 68.37.Ps; 81.16.Nd; 68.47.Mn

Keywords: Atomic force microscopy; Water meniscus; Electrostatic forces

\section{Introduction}

The formation of structures that are an order of magnitude larger $(10-80 \mathrm{~nm})$ than the distance between an atomic force microscope (AFM) tip and various surfaces in response to an applied electrostatic potential have yet to receive an adequate physical explanation, even though such features have been reported on polymer, silicon, and metal surfaces. In some manifestations of scanning probe microscopy (SPM) when a bias is applied between tip, and substrate, the height of the feature formed in the substrate substantially exceeds the initial tip-surface separations - at times by over an order of magnitude and larger. This has been observed for diverse systems including semiconductors [1,2] and recently in polymers $[3,4]$ including conductive polymers [5], but the origin of

\footnotetext{
*Corresponding author. Tel.: + 1330 9728356; fax: + 13309726918.

E-mail address: sfl@physics.uakron.edu (S.F. Lyuksyutov).

${ }^{1}$ Current address: School of Chemistry and Biochemistry, Georgia Institute of Technology, Atlanta, GA 30332, USA.
}

the force leading to tip retraction has not been adequately accounted for. To understand this peculiar SPM tip behavior is important on the reasons of nanofabrication, surface characterization based on SPM [6], and also for instrumental development of novel AFM systems.

The goal of this work is to study the nature of AFM tipdielectric spontaneous repulsion through the balance of forces involved in this process, and to identify the key factors responsible for this process. Complete understanding of the process is complicated by the fact that the specific spatial details of the tip-surface contact profile as well as cantilever motion with applied bias is exceedingly difficult to observe and quantify. Although in this study we primarily concentrate on experimental aspect of this process in polymers, the associated conclusions are generally applicable to bio-macromolecules and selfassembled monolayers. We shall analyze temporal behavior of AFM tip through the vertical deflection transients. The features are patterned in polymeric surface through nanoscopic soft condensed matter transport with a biased AFM tip retraction from the surface. The height of the 
features is determined by a magnitude of tip's retraction thus presenting a degree of AFM tip mechanical displacement in close vicinity (several nanometers) of nonconductive dielectric surface.

\section{Experiment}

A commercial VEECO Digital Instruments 3100 Dimensions AFM with Nanoscope IV controller was used to study tip' erratic behavior. When voltage is applied to the tip dwelling $0.5-5 \mathrm{~nm}$ above the dielectric (polymeric film spun-coated on conductive $\mathrm{Au}-\mathrm{Pd}$ substrate), an electric field of the order of magnitude $10^{8}-10^{9} \mathrm{Vm}^{-1}$ is induced in tip-surface junction. The electric field between the tip and a conductive substrate initiates current flow through the film in the range $100 \mathrm{pA}$ to $100 \mathrm{nA}$ (monitored by Keithley 6485 picoammeter). This current heats polymer molecules above the glass transition temperature, facilitating the flow of polarized melt towards the negatively biased tip. Nanoscale features are formed in the surface as a result of this interaction between tip and polymer and remain frozen after the process completed. The height of the features may serve as a parameter for analysis of the tip' lift-up from the surface.

Fig. 1 depicts typical raised nanostructures in three polymer films. In brief, these representative features: (a): 25-30 nm (b): $15 \mathrm{~nm}$; (c): $32 \mathrm{~nm}$ all on poly-(methylmethacylate) (PMMA); (d): 5-20 nm; (e): 15-25 nm on polystyrene (PS); (f): $85 \mathrm{~nm}$ on fluorinated poly (benzoxazole) (PBO) were created. All the structures are greater than tip-surface separation in contact mode, which varies between fractions of, to several nanometers. Relative humidity affects the height because the radius of the water meniscus around the tip impacts the feature formation processes [7,8].

We have studied experimentally the tip temporal behavior under applied electric voltage. The analog signal from AFM photodetector corresponding to the vertical deflection of the cantilever was monitored with Tektronix TDS 220 oscilloscope (1GS s${ }^{-1}$ sampling rate). The $\mathrm{C}++$ script linked to the AFM controlling software was used to synchronize the oscilloscope, and to transfer the captured deflection transients to the computer. Fig. 2 presents the temporal dependence of the tip deflection, which is representative of the various features of different height formed in PS. The negatively biased AFM cantilever retracts from the surface during the time $\tau_{2}=45 \mu$ s and then bends towards the surface during time $\tau_{1}=145 \mu \mathrm{s}$. The oscillating background of the deflection signal is related to thermal vibrations of the cantilever. Consider that the applied voltage charges two capacitors, one corresponding to the tip-substrate and the other to the cantileversubstrate (see inset Fig. 2). The charging time $(\tau=\mathrm{RC})$ will be proportional to the capacitance of each capacitor. Accounting for the geometry of a triangular lever, height of the tip $(15 \mu \mathrm{m})$, and the area under the tip apex $\left(3.8 \times 10^{3} \mathrm{~nm}^{2}\right)$, the ratio of cantilever-substrate $\left(C_{1}\right)$ to tip-substrate $\left(C_{2}\right)$ capacitances is estimated to be 3.7

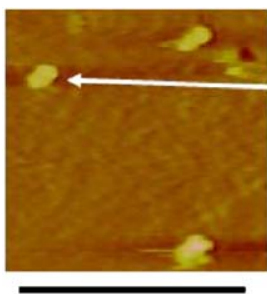

(a) $1 \mu \mathrm{m}$

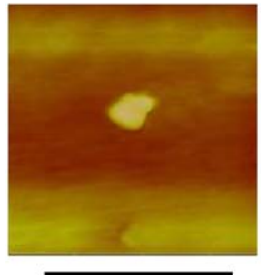

(b) $600 \mathrm{~nm}$

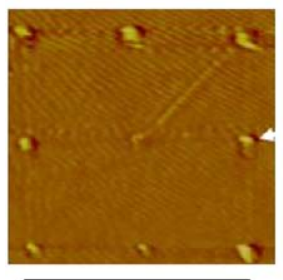

(d) $\mathbf{2} \boldsymbol{\mu m}$

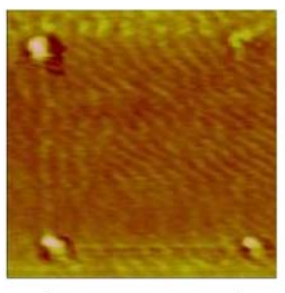

(e)
$1 \mu \mathrm{m}$

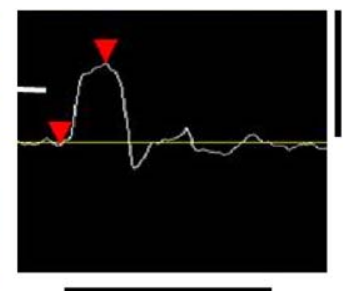

$300 \mathrm{~nm}$

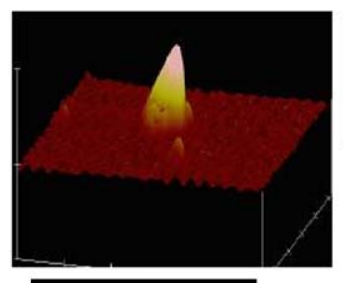

(c) $1 \mu \mathrm{m}$

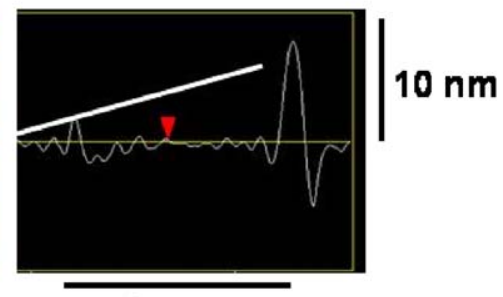

$1 \mu \mathrm{m}$

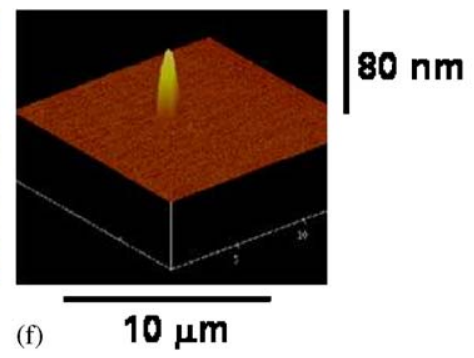

Fig. 1. AFMEN patterned structures at relative humidity varied between 40-50\%: (a) Three raises in 50-nm thick PMMA $\left(M_{\mathrm{W}} \sim 525 \mathrm{k}\right)$ of $25 \mathrm{~nm}$ high and $120 \mathrm{~nm}$ wide (bias voltage $-18 \mathrm{v}$, exposure time $0.5 \mathrm{~s}$, current $-300 \mathrm{nA}$ ), $27 \mathrm{~nm}$ high and $135 \mathrm{~nm}$ wide (bias voltage $-20 \mathrm{v}$, exposure time $0.5 \mathrm{~s}$, current $-320 \mathrm{nA}$ ), and $30 \mathrm{~nm}$ high and $150 \mathrm{~nm}$ wide (bias voltage $-23 \mathrm{v}$, exposure time $0.5 \mathrm{~s}$, current $-350 \mathrm{nA}$ ); (b) a single raise patterned in PMMA of $15 \mathrm{~nm}$ height and $100 \mathrm{~nm}$ width at $-20 \mathrm{~V}$ at exposure time $1 \mathrm{~s}$; (c) $32 \mathrm{~nm}$ high and $75 \mathrm{~nm}$ wide in $35 \mathrm{~nm}$ thick PMMA $\left(M_{\mathrm{W}} \sim 980 \mathrm{k}\right)$ (bias voltage $-25 \mathrm{~V}$ at exposure time $1 \mathrm{~s}$ ); (d) eight raises of height varied between 5 and $20 \mathrm{~nm}$ (bias voltage varied between -14 and $-16 \mathrm{~V}$ at $-0.4 \mathrm{~V}$ step, the average current $\sim-60 \mathrm{nA}$ ); (e) Four raises of height varied between 15 and $25 \mathrm{~nm}$ (bias voltage varied between -17 and $-20 \mathrm{~V}$ at $-0.5 \mathrm{~V}$ step, the average current $\sim-70 \mathrm{nA}$ ) formed in $35-\mathrm{nm}$ thick PS $\left(M_{\mathrm{W}} \sim 110 \mathrm{k}\right)$ film; (f) Anomalously high raise of $85 \mathrm{~nm}$ height and $150 \mathrm{~nm}$ width patterned in $6 \mathrm{FPBO}$ at $-30 \mathrm{~V}$ and $1 \mathrm{~s}$;. Polymer films of roughness less than $1 \mathrm{~nm}$ were spun coated onto conductive $\mathrm{Au}-\mathrm{Pd}$ film co-sputtered on silicon wafer. AFM tips used in this study were highly conductive tungsten carbide coated K-TEK tips with the apex radius of $30-40 \mathrm{~nm}$.

comparable to the experimental ratio of deflection times $\tau_{1} / \tau_{2}$. Thus the tip-substrate capacitor is quickly charged and the initial deflection is directly associated with this interaction. At longer times, the larger capacitance of the 


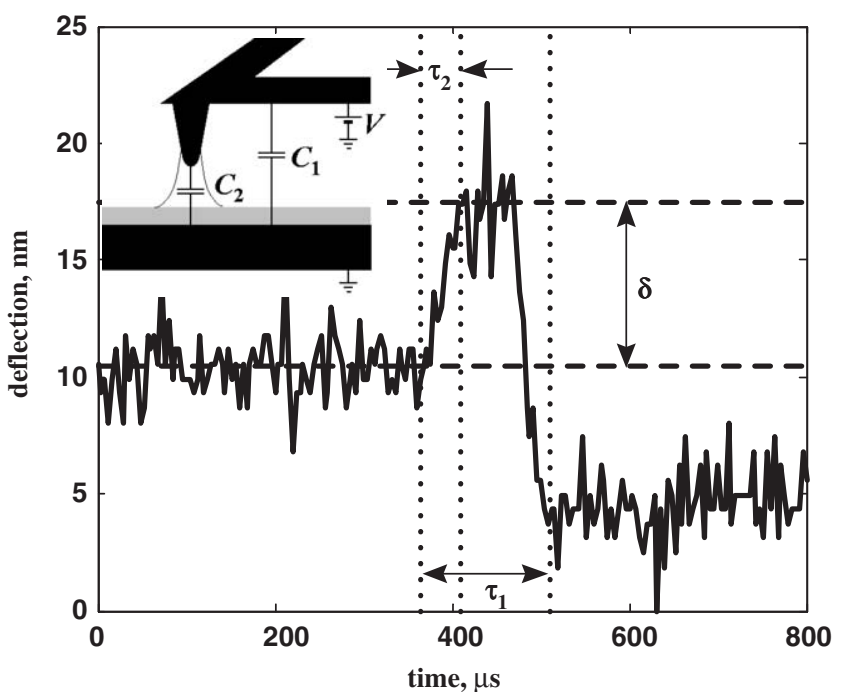

Fig. 2. A representative temporal dependence of the tip deflection from $30 \mathrm{~nm}$ thick PS film $\left(M_{\mathrm{W}} \sim 110 \mathrm{k}\right)$. The tip was biased at $-41 \mathrm{~V}$ for $280 \mu \mathrm{s}$ using rectangular pulse shape starting at $360 \mu$ s as indicated by the dotted vertical line. The rise time of the voltage source is below $5 \mu$ s. Inset: a schematic presentation of the two-capacitor system formed by tip-dielectric-conductor, and lever-dielectric-conductor layers.

cantilever-substrate dominates, leading to an attraction between the negatively charged lever and positive substrate, and associated reduction in the vertical deflection signal.

\section{Results and discussion}

The original explanation of an abrupt tip deflection was based on the fact that in AFM constant force mode, the enabled feedback retracted the AFM tip from the surface allowing the polymer melt to flow toward the biased tip forming unusually high nanostructures. However this still implies the raising polymer must exert force on the tip to cause deflection so that the feedback loop engages tip retraction. The features form also when the AFM feedback loop is disabled and the tip "pulls itself up" from softened polymer surface. The key question is what is the source of the initial spontaneous tip retraction (during time $\left.\tau_{2}=45 \mu \mathrm{s}\right)$ when a bias is applied across the gap?

We are concerned with the terms contributing to the net force $F$ between a biased tip and a dielectric surface: $F=F_{\mathrm{E}}+F_{\mathrm{W}}+F_{\mathrm{M}}$. Here $F_{\mathrm{E}}$ and $F_{\mathrm{W}}$ present the electrostatic and Van der Waals contributions, respectively, and $F_{\mathrm{M}}$ collects the contributions related to the water meniscus. The analytical solution based on the method of images for the electrostatic problem comprising an electrically biased tip and a thin dielectric film [9] provides the expression for electrostatic term $F_{\mathrm{E}}$ calculated from the image charge distribution in the following form:

$F_{\mathrm{E}}=\pi \varepsilon_{0} V^{2} R^{2}\left(\frac{\eta}{h^{2}}-\left(1-\eta^{2}\right) \sum_{n=0}^{\infty} \frac{\eta^{n}}{(a(n+1)+h)^{2}}\right)$.
Here, $V$ is the bias voltage, $a$ is the polymer film thickness, $h=t+R, t$ is the tip-surface distance, $R$ is the tip radius, and $\eta=\left(\varepsilon_{2}-\varepsilon_{1}\right) /\left(\varepsilon_{2}+\varepsilon_{1}\right)$, with $\varepsilon_{1}$ as the dielectric constant of the polymer film and $\varepsilon_{2}$ as the effective dielectric constant of the water meniscus, which is a function of the electric field because of dielectric saturation. For small fields the dielectric response of water is linear with $\varepsilon_{2}=80$. The dielectric saturation becomes substantial as the field grows stronger than $10^{7}-10^{8} \mathrm{Vm}^{-1}$ resulting in decrease of $\varepsilon_{2}$ with the field down to value of 2 . The molecular dynamics simulations provide insight into dielectric saturation phenomena [10]. We treat $\varepsilon_{2}$ as an effective constant for the calculation of electrostatic force. The two limiting cases of (1) are of interest. In thick dielectric film at small tip-surface separation, the expression (1) is reduced to:

$\left.F_{\mathrm{E}}\right|_{h \ll a}=\frac{1}{4 \pi \varepsilon_{0}} \frac{\eta Q^{2}}{4 h^{2}}$.

This force corresponds to the interaction between the charges $Q$ and $\eta Q$, where the charge $Q=4 \pi \varepsilon_{0} R V$, is located at the distance $h$ above and below the boundary of a semi-infinite dielectric layer $\varepsilon_{1}$. The force is repulsive $\left(\varepsilon_{2}>\varepsilon_{1}, \eta>0\right)$, implying the tip retracts from a dielectric surface at small separations $t$. In the opposite limit, when the tip-surface separation $t$ exceeds significantly the dielectric film thickness $a$, expression (1) is reduced to

$\left.F_{\mathrm{E}}\right|_{h \gg a}=-\frac{1}{4 \pi \varepsilon_{0}} \frac{Q^{2}}{4 h^{2}}$,

indicating on the long-range electrostatic attraction between the biased tip and the conductive substrate.

Fig. 3 depicts the variation of the electrostatic force $F_{\mathrm{E}}$ with respect to $t$ for the different values of $\varepsilon_{2}$. It clearly suggests a dominant electrostatic repulsion at close

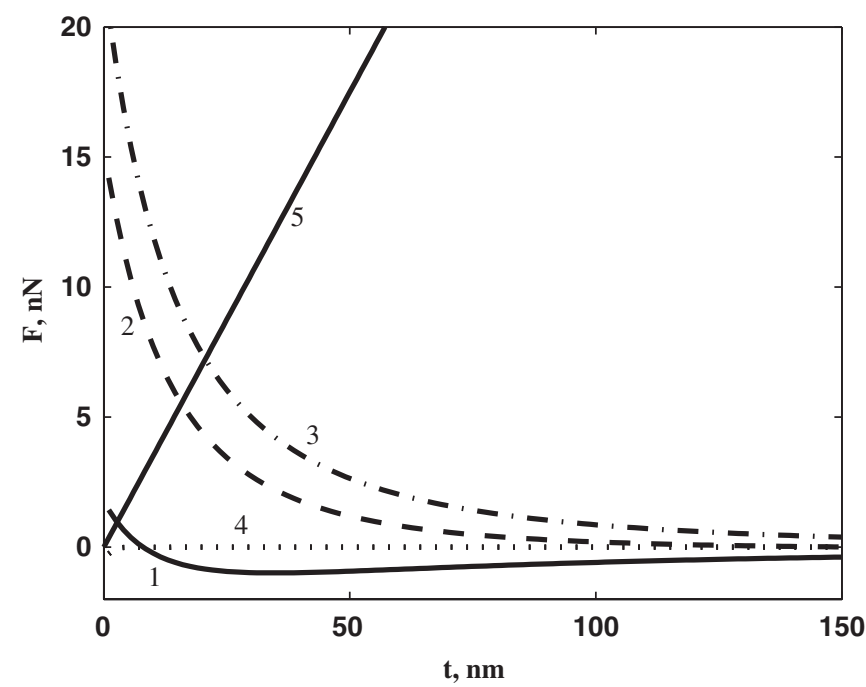

Fig. 3. The variation of the electrostatic $F_{\mathrm{E}}$ (curves 1-3) and Van der Waals $F_{\mathrm{W}}$ (curve 4 ) forces with respect to $t$. The typical values were used: the tip's radius $R=30 \mathrm{~nm}$; the polymer film thickness $a=R$; the bias voltage $V=-30 \mathrm{~V} ; \varepsilon_{1}=2.5$ (PS). The values of $\varepsilon_{2}$ were: 5 (1) solid line; 20 (2) dashed line; 80 (3) dash-dot line. The line 5 corresponds to the bending force of the cantilever at the spring constant of $0.35 \mathrm{Nm}^{-1}$. 
tip-sample separations (less than $50 \mathrm{~nm}$ ) for the whole range of the admissible $\varepsilon_{2}$ values. The Van der Waals attractive force, $F_{\mathrm{W}}$, acting between the flat sample surface and the tip of radius $R$ separated by the distance $t$, is given by: $F_{\mathrm{W}}=-A R / 12 t^{2}$. The value of the Hamaker constant $A$ for condensed matter is typically in the order of $10^{-19} \mathrm{~J}$, corresponding to the negligibly small contribution (presented as curve 4 in Fig. 4) in comparison to the electrostatic terms.

The free energy analysis for the system comprising a biased AFM tip, water meniscus, and dielectric surface suggests [11] that $F_{\mathrm{M}}$ is dominated by water polarization in the external electric field. The force $F_{\mathrm{M}}$ is repulsive: water tends to condense in the tip-surface junction by increasing $t$ and lowering the free energy of the system [11]. The total tip-sample repulsive force results in the cantilever bending away from the surface thus establishing the tip-sample

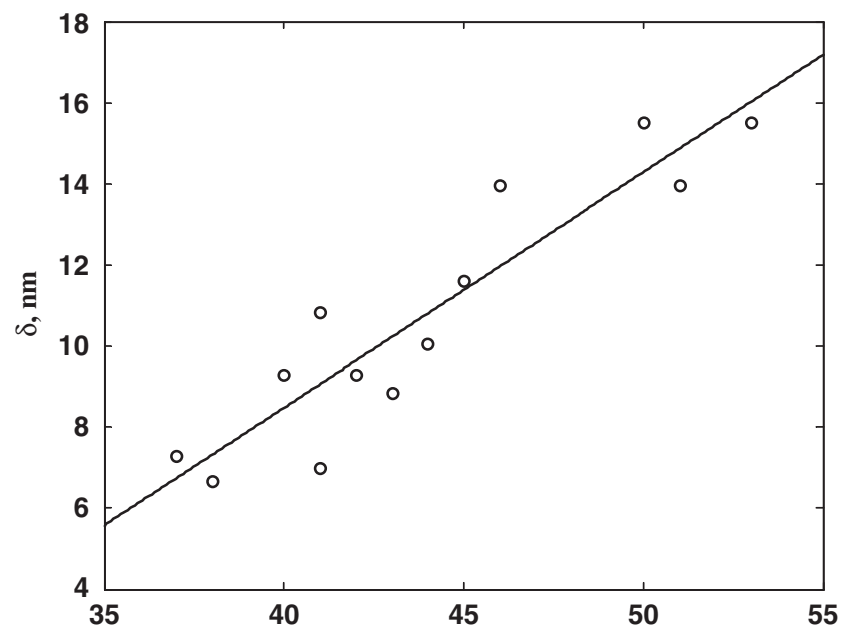

(a)

Bias voltage, $\mathrm{V}$

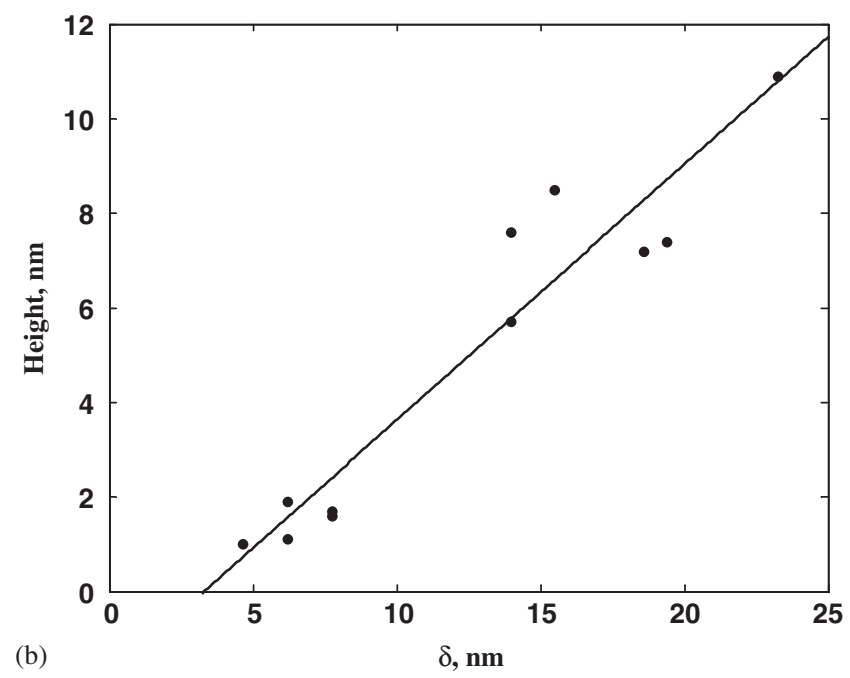

Fig. 4. Experimental dependencies of: (a) the deflection factor $\delta$ as a function of the bias voltage $V$; (b) nanostructure height patterned in polymer film versus deflection factor $\delta$ for PS $(30 \mathrm{~nm}$ thick film, $\left.M_{\mathrm{W}} \sim 110 \mathrm{k}\right)$. The values of $\delta$ were determined from the deflection signal transients presented in Fig. 2. separation $t$ comparable to the tip's radius. The value of $t$ depends on parameters such as bias voltage $V$, dielectric constants $\varepsilon_{1}$, and $\varepsilon_{2}$, polymer film thickness $a$, and cantilever spring constant. The analysis [11] also suggests that $t$ mostly depends on the tip bias $V$ and cantilever spring constant. The variations of $t$ with respect to the dielectric constants, and polymer film thickness found to be less pronounced.

The free energy analysis accounting both electrostatic and meniscus contributions, $F_{\mathrm{E}}$ and $F_{\mathrm{M}}$, predicts a nearly linear increase of the tip deflection with the bias voltage. Fig. 4 presents an experimental dependence of the deflection factor $\delta$ on the tip's bias voltage $V$ for $30 \mathrm{~nm}$ thick PS film. The linear trend agrees well with the calculation [11]. The height of the patterned nanostructures, in turn, was found to be linearly proportional to the deflection factor $\delta$. It should be noted that the observed trend in topographical height is related indirectly to the variation of the tip deflection parameter $\delta$ [12].

\section{Summary}

There are three outcomes from this consideration.

The first outcome is the physical explanation of biased AFM tip' deflection from dielectric surfaces. The analysis of the forces in the system indicates that the equilibrium distance is comparable to the tip's radius. It suggests that the spontaneous tip lift-up is associated with the tip' electrostatic repulsion from the surface in the doublelayered (water and polymer) configuration. Another reason is related to the volume of water penetrating in the tip-surface junction. Thus the peculiar behavior of the AFM tip can be attributed to energy transfer from electrostatic field to the potential energy of AFM cantilever.

The second outcome is a simple method for resolving temporal behavior of the system comprising AFM tip and cantilever. The experimental data collected in this work indicate initially fast (shorter than $50 \mu \mathrm{s}$ ) deflection of the biased tip away from the surface. A spontaneous lift-up of the tip has been monitored experimentally and found to be proportional to the height of nanostructures raised in films. The method allows investigate a real tip-surface dynamical response separately from the response associated mainly with electrostatic attraction between AFM lever and arbitrary surfaces.

The third outcome of this study is practical aspect of the effect of tip' abrupt retraction for nanosructures formation in dielectrics for data storage applications. The data storage in this century will apparently be based on magnetism but in several decades an existing magnetic data storage technology will be pushed to the limit. The alternatives to magnetic data storage could become different media (such as polymers), and techniques (such as holographic methods). For polymers, the ability to reshape the surface topography, in contrast to removing or depositing material, has created possible alternatives to 
magnetic-based technologies for multiple read-write storage media. The majority of polymer-patterning techniques are based on the spatially selective removal, formation or deposition of polymers. Our technique: AFMEN [3,4] (atomic force microscopy-assisted (AFM) electrostatic nanolithography) generates features by mass transport of polymer without chemical cross-linking, polymer degradation, or ablation.

\section{Acknowledgements}

S.F.L. and P.B.P acknowledge support from AFOSR/ AFRL and in the frames of STW-21, grant FA 9550-05-10471-3 Program. S.F.L. wishes to thank Materials and Manufacturing Directorate (MLBP) for the opportunity to perform part of this project at WPAFB, OH. Authors are grateful to Dr. G. Sigalov for valuable discussions.

\section{References}

[1] E.S. Snow, P.M. Campbell, Science 270 (1995) 1639.

[2] H. Kuramochi, K. Ando, H. Yokoyama, Surf. Sci. 542 (2003) 56.
[3] S.F. Lyuksyutov, R.A. Vaia, P.B. Paramonov, S. Juhl, L. Waterhouse, R.M. Ralich, G. Sigalov, E. Sancaktar, Nature Mater. 2 (2003) 468.

[4] S.F. Lyuksyutov, R.A. Vaia, P.B. Paramonov, S. Juhl, Appl. Phys. Lett. 83 (2003) 4405.

[5] S.-Y. Jang, M. Marquez, G.A. Sotzing, J. Am. Chem. Soc. 126 (2004) 9476.

[6] M.A. Poggi, E.D. Gadsby, L.A. Bottomley, W.P. King, E. Oroudjev, H. Hansma, Anal. Chem. 76 (2004) 3429.

[7] J. Jang, G.C. Schatz, M.A. Ratner, J. Chem. Phys. 116 (2002) 3875.

[8] S.F. Lyuksyutov, P.B. Paramonov, I. Dolog, R.M. Ralich, Nanotechnology 14 (2003) 716.

[9] S.F. Lyuksyutov, P.B. Paramonov, R.A. Sharipov, G. Sigalov, Phys. Rev. B 70 (2004) 174110.

[10] G. Sutmann, J. Electroanal. Chem. 450 (1998) 289.

[11] P. B. Paramonov, S. F. Lyuksyutov, R. A. Vaia, arxiv.org: cond-mat/ 0505457.

[12] While the initial tip retraction away from the polymer surface provides room for a polymer nanostructure to grow, the resulting nanostructure height is determined by interplay of the following factors. Surface tension accompanying polymer deformation competes against electrostatic ponderomotive force pulling polarized polymer upward. This interplay limits the growth of the nanostructure. Additionally, a certain extent of the shape relaxation takes place as deformed polymer cools down below $T_{\mathrm{g}}$. As a result, the relation between the tip deflection factor $\delta$, and topographical height has indirect character. 